\title{
Optimum SINR Receiver in Dispersive CDMA Channels
}

\author{
S. Chen and A.G. Constantinides \\ Communications and Signal Processing Group \\ Department of Electrical and Electronic Engineering \\ Imperial College of Science, Technology and Medicine, London SW7 2BT, UK \\ Email: shujun.chen@ic.ac.uk
}

\begin{abstract}
In this paper, we address the problem of blind channel estimation and interference suppression for directsequence code division multiple access (DS-CDMA) systems over frequency-selective fading channels. The approach taken is based on our recently published work on the autocorrelation of contribution matrix (ACM) algorithm. We demonstrate that the receiver developed on the basis of the estimated channel through the ACM algorithm is asymptotically optimal in the sense that it maximizes the output signal to interference and noise ratio (SINR). A significant advantage of the proposed algorithm is that it tolerates both synchronization error in the desired user and multipath delay spread overestimation over a wide range of values.
\end{abstract}

\section{INTRODUCTION}

Direct sequence code division multiple access (DS-CDMA) techniques have established their dominant position as the basis for the realization of the physical layer in most third generation wireless cellular networks. Compared with traditional time or frequency division multiple access (TDMA/FDMA) techniques, the CDMA approach exhibits numerous advantages including spectral efficiency, anti-jamming capability, flexibility and other positive features [1].

The conventional approach of using matched filters in a nonorthogonal CDMA system suffers from the near-far problem. Recently considerable research effort has been concentrated on the design of multi-user detectors, such as the optimal maximum likelihood detector, the decorrelator, the minimum mean square error (MMSE) detector [2], the minimum output energy (MOE) detector [3] and the subspace detector [4]. In the development of these algorithms the assumption is made that the multiplexed signals transmit through frequency flat fading channels. However, in the current third generation communication systems and beyond, because of the request for high dataaccess rates for mobile users, the CDMA signal bandwidth is much wider than the channel coherence bandwidth [5]. The effect of distortion on the signature waveforms and the intersymbol interference (ISI) present due to the multipath nature of wireless channels cannot be neglected. Therefore, the received signature waveforms or the information on the channel state must be obtained before any multiuser detection techniques mentioned above can be applied. Although a training-based scheme can accomplish this task, the transmission of training symbols significantly reduces channel efficiency.
Driven by the above needs the problem of blind channel estimation has received significant research interest over the last decade or so. Initial and significant steps are taken in [6], [7] and [8]. In these papers, the developed algorithms require the processing of a large number of samples and large size matrices. Unfortunately, such demands lead not only to high computational requirements but also to long delays in information symbol detection. In recently published work for simplification in algorithm development, only the non-ISI part of the received signal is considered. Under these circumstances since the energy in the ISI part of signal replica is not explored, the algorithms developed so far suffer from performance degradation especially when the multipath delay spread is large. An important improvement to the above is proposed by $\mathrm{T}$ satsanis and $\mathrm{Xu}$ in [9]. Indeed, by exploiting all of the received signal energy, the authors develop the Minimum Variance (MV) algorithm. While this is a significant advance from earlier approaches, any mismatch in channel estimation, which is bound to be present in any circumstances, will degrade the performance of the consequent MMSE receiver.

In this present paper we consider the problem of blind channel estimation and interference suppression for asynchronous DS-CDMA systems over frequency dispersive channels from a perspective based on our earlier work. Specifically we study the performance of the autocorrelation of contribution matrix (ACM) algorithm we proposed recently in [10]. In particular, we show that this new blind algorithm outperforms the MV algorithm in [9] almost without increasing computational complexity and provides a performance very close to the nonblind MMSE algorithm.

\section{SYSTEM MODEL}

Let us consider a CDMA uplink system model in which $\mathrm{K}$ users simultaneously and asynchronously transmit a binary phase-shift keying (BPSK) signal. The complex envelope of the received signal is written as

$$
y(t)=\sum_{j=-\infty}^{+\infty} \sum_{k=1}^{K} A_{k} b_{k}(j) s_{k}\left(t-\tau_{k}-j T_{s}\right) * p_{k}(t)+v(t)
$$

where $s_{k}(t), \tau_{k}$ and $A_{k}$ denote the transmitter signature waveform, the delay and the received amplitude of user $k$, respectively. The quantity $b_{k}(j) \in\{+1,-1\}$ denotes the 
BPSK information symbol transmitted by user $k$ at the $j$ th interval, $T_{s}$ denotes the symbol interval, and $v(t)$ denotes white Gaussian noise with power $\sigma^{2}$. The quantity $p_{k}(t)$ represents the impulse response of the propagation channel of user $k$ with maximum delay spread as $T_{m}$. In this paper we assume that the channel fading varies slowly so that $p_{k}(t)$ can be regarded as stationary over a large number of symbol intervals. Further let $T_{c}$ denote the chip duration, $L=T_{s} / T_{c}$ the system spreading gain, $\left\{c_{k}(n)\right\}_{n=0}^{n=L-1}$ the spreading sequence assigned to user $k$, and $\varphi(t)$ the unitenergy chip waveform with support only on $\left[0, T_{c}\right]$, so that

$$
\begin{aligned}
h_{k}(t) & =A_{k} s_{k}\left(t-\tau_{k}\right) * p_{k}(t) \\
& =A_{k} \sum_{m=0}^{L-1} c_{k}(m) g_{k}\left(t-m T_{c}\right),
\end{aligned}
$$

where $h_{k}(t)$ is the received signature waveform of user $\mathbf{k}$ and $g_{k}(t)=\varphi\left(t-\tau_{k}\right) * p_{k}(t)$ is the unknown received chip waveform. Substituting (2) into (1), we obtain

$$
y(t)=\sum_{k=1}^{K} \sum_{j=-\infty}^{+\infty} b_{k}(j) h_{k}\left(t-j T_{s}\right)+v(t) .
$$

Without loss of generality we assume that the user of interest is user 1 . In the methods we study below the system is assumed to have been coarsely synchronized with the desired user such that an observation window of duration $T_{s}+T_{w}$ can cover the complete duration of $h_{1}(t)$. This assumption implies that the synchronization error $\tau_{1}$ is restricted to be between 0 and $T_{w}-T_{m}$. Such synchronization error in timing acquisition may also be ascribed to a relative position change between the base station and mobile users after timing acquisition is performed. Alternatively $T_{w}$ can also be regarded as an estimation of the multipath delay spread at the receiver. Therefore, this model takes both synchronization error and multipath delay spread over estimation into consideration, degradations which are usually encountered in practice. Now we convert the observations within the window to a discrete model by passing $y(t)$ through an integrator and sampling the output at a rate of $M$ samples per chip. Let $L+L_{w}-1=\left(T_{s}+T_{w}\right) / T_{c}$ denote the total number of chips in one observation window, $y(n)$ collect all the sampling outputs as $[y(M L n), \ldots, y(M L n+$ $\left.\left.M\left(L+L_{w}-1\right)-1\right)\right]^{T}$ with superscript $T$ denoting vector transpose and $\mathbf{v}(n)$ collect the discrete noise, we have

$$
\mathbf{y}(n)=b_{1}(n) \mathbf{h}_{1,0}+\sum_{k=1}^{K} \sum_{j \in \Omega_{k}} b_{k}(n+j) \mathbf{h}_{k, j}+\mathbf{v}(n)
$$

where the set $\Omega_{1}$ includes the ISI symbols of user 1 falling in the observation window, and similarly the sets $\Omega_{k}(k=$ $2, \ldots, K)$ include the multiple access interference (MAI) symbols of each interfering user. In this paper, upper and lower case bold letters denote matrices and vectors. We refer to $\mathbf{h}_{1,0}$ as the "contribution vector" of symbol $b_{1}(n)$ and use this term in the rest of this paper. After some mathematical manipulation we can obtain

$$
\mathbf{h}_{1,0}=\mathbf{C}_{1} \mathbf{g}_{1}
$$

where $\mathrm{g}_{1}$ denotes the discrete version of $g_{1}(t)$ and $\mathbf{C}_{1}$ is a $M\left(L+L_{w}-1\right) \times M L_{w}$ block Toeplitz matrix defined as

$$
\mathbf{C}_{1} \triangleq\left[\begin{array}{cccc}
c_{1}(0) \mathbf{I}_{M} & & & \\
c_{1}(1) \mathbf{I}_{M} & c_{1}(0) \mathbf{I}_{M} & & \\
\vdots & c_{1}(1) \mathbf{I}_{M} & & \\
& \vdots & \ddots & c_{1}(0) \mathbf{I}_{M} \\
c_{1}(L-1) \mathbf{I}_{M} & \vdots & c_{1}(1) \mathbf{I}_{M} \\
& c_{1}(L-1) \mathbf{I}_{M} & & \vdots \\
& & & c_{1}(L-1) \mathbf{I}_{M}
\end{array}\right],
$$

where $\mathbf{I}_{M}$ is an identity matrix of order $M$. It is worth noting that $\mathbf{g}_{1}$ has combined all the necessary information to reconstruct the received signature waveform including the transmitter, channel and receiver impulse response as well as the delay $\tau_{1}$. Thus, the problem of receiver signature waveform estimation has been transformed to the estimation of $\mathbf{g}_{1}$. The forms of contribution vectors $\mathbf{h}_{1, j}$ of all ISI symbols and $\mathbf{h}_{k, j}$ of all MAI symbols are similar and thus omitted here. Finally, let $\mathbf{H}_{M A I}$ contain all these interfering contribution vectors, and $\mathbf{b}_{M A I}(n)$ contain all the interfering information symbols, so that we obtain the more compact form (4) as

$$
\mathbf{y}(n)=b_{1}(n) \mathbf{C}_{\mathbf{1}} \mathbf{g}_{1}+\mathbf{H}_{M A I} \mathbf{b}_{M A I}(n)+\mathbf{v}(n) .
$$

\section{Algorithm DeVelopment}

Before we introduce the ACM algorithm we first review the MMSE algorithm so as to build a basis for the derivation of the ACM algorithm. Given a knowledge of the channel state $\mathbf{g}_{1}$, the MMSE receiver [9] is given by

$$
\mathbf{w}_{M M S E}=\mathbf{R}_{\mathbf{y}}^{-1} \mathbf{C}_{1} \mathbf{g}_{1},
$$

where $\mathbf{R}_{\mathbf{y}}=E\left\{\mathbf{y}(n) \mathbf{y}^{H}(n)\right\}$ denotes the correlation matrix of the received signal. We can rewrite the MMSE receiver into a form of maximizing the signal to interference-and-noise ratio (MSINR). Let $P=\mathbf{w}^{H} \mathbf{R}_{\mathbf{y}} \mathbf{w}$ denote the overall receiver output power and $P_{b_{1}(n)}=\mathbf{w}^{H} \mathbf{h}_{1,0} \mathbf{h}_{1,0}^{H} \mathbf{w}$ denote the output power of the desired symbol $b_{1}(n)$, the output $S I N R$ is then defined as $\frac{P_{b_{1}(n)}}{\left(P-P_{b_{1}(n)}\right)}$. It is evident that the receiver based on the MSINR criterion can be written as

$$
\mathbf{w}_{M S I N R}=\arg \max _{\mathbf{v}} \frac{\mathbf{v}^{H} \mathbf{h}_{1,0} \mathbf{h}_{1,0}^{H} \mathbf{v}}{\mathbf{v}^{H} \mathbf{R}_{\mathbf{y}} \mathbf{v}},
$$

where superscript $H$ denotes vector Hermitian transpose. Due to the equivalence between the MMSE criterion and the MSINR criterion, the solutions of (7) and (8) must be identical. By substituting (5) and (7) into (8) we obtain the following alternative form of the MMSE receiver

$$
\mathbf{w}_{M M S E}=\mathbf{R}_{\mathbf{y}}^{-1} \mathbf{C}_{1} \hat{\mathbf{g}}_{1}, \hat{\mathbf{g}}_{1}=\frac{\mathbf{v}^{H} \mathbf{C}_{1}^{H} \mathbf{R}_{\mathbf{y}}^{-1} \mathbf{R}_{b_{1}} \mathbf{R}_{\mathbf{y}}^{-1} \mathbf{C}_{1} \mathbf{v}}{\mathbf{v}^{H} \mathbf{C}_{1}^{H} \mathbf{R}_{\mathbf{y}}^{-1} \mathbf{C}_{1} \mathbf{v}}
$$

where $\hat{\mathrm{g}}_{1}$ denotes an estimation of the channel of user 1 and $\mathbf{R}_{b_{1}}=A_{1}^{2} \mathbf{C}_{1} \mathbf{g}_{1} \mathbf{g}_{1}^{H} \mathbf{C}_{1}^{H}$ can be regarded as the subspace matrix of the desired symbol $b_{1}(n)$. The expressions in (9) are 
not feasible in their present form for algorithm development since the channel estimation requires explicit knowledge of the unknown vector $\mathbf{g}_{1}$. Nevertheless they do provide a new framework to deal with the problem of channel estimation which with judicious restructuring leads to the realizable ACM algorithm as indicated in the following.

On substituting the matrix $\mathbf{R}_{b_{1}}$ in (9) by the complete signal subspace $\mathbf{R}_{b}=\mathbf{h}_{1,0} \mathbf{h}_{1,0}^{H}+\mathbf{H}_{M A I} \mathbf{H}_{M A I}^{H}$, we obtain the new ACM algorithm, given by

$\mathbf{w}_{A C M}=\mathbf{R}_{\mathbf{y}}^{-1} \mathbf{C}_{1} \hat{\mathbf{g}}_{1}, \hat{\mathbf{g}}_{1}=\arg \max _{\|\mathbf{v}\|=1} \frac{\mathbf{v}^{H} \mathbf{C}_{1}^{H} \mathbf{R}_{\mathbf{y}}^{-1} \mathbf{R}_{b} \mathbf{R}_{\mathbf{y}}^{-1} \mathbf{C}_{1} \mathbf{v}}{\mathbf{v}^{H} \mathbf{C}_{1}^{H} \mathbf{R}_{\mathbf{y}}^{-1} \mathbf{C}_{1} \mathbf{v}}$

The matrix $\mathbf{R}_{b}$ above includes not only the subspace of the desired symbol but also the subspaces of all interfering symbols. Thus based on a superficial examination it would seem that the ACM algorithm is unconvincing. However, we show in next section that under a regular identifiability condition the new algorithm is able to estimate the channel, and to provide, in the asymptotic sense, a performance identical to the MMSE algorithm. In the rest of this section we present an alternative form of the ACM algorithm and show that it can be implemented blindly. Substituting $\mathbf{R}_{b}=\mathbf{R}_{y}-\sigma^{2} \mathbf{I}$ into the second equation in (10) and with some mathematical manipulations, we can present the alternative form of ACM algorithm as,

$\mathbf{w}_{A C M}=\mathbf{R}_{\mathbf{y}}^{-1} \mathbf{C}_{1} \hat{\mathbf{g}}_{1}, \hat{\mathbf{g}}_{1}=\arg \min _{\|\mathbf{v}\|=1} \frac{\mathbf{v}^{H} \mathbf{C}_{1}^{H} \mathbf{R}_{\mathbf{y}}^{-1} \mathbf{R}_{\mathbf{y}}^{-1} \mathbf{C}_{1} \mathbf{v}}{\mathbf{v}^{H} \mathbf{C}_{1}^{H} \mathbf{R}_{\mathbf{y}}^{-1} \mathbf{C}_{1} \mathbf{v}}$.

The spreading code of the desired user is always available at the receiver and $\mathbf{R}_{\mathbf{y}}$ can be estimated from the received signal. Clearly the ACM algorithm requires no more information and can thus be implemented blindly.

\section{Performance Analysis of the ACM Algorithm}

In this section, we examine the performance of the ACM algorithm and compare it to that of the MMSE algorithm. It is immediately observed on comparing (7) and (11) that the proposed ACM receiver and the well known MMSE receiver produce identical interference suppression. There is, however, one fundamental and significant difference. The ACM algorithm uses the estimated channel, while the MMSE algorithm requires the information of channel state as a prior knowledge. Therefore, the performance comparison between these two algorithms focuses on quantifying the mismatch between the estimated channel $\hat{\mathbf{g}}_{1}$ and its accurate counterpart $\mathbf{g}_{1}$

\section{A. Derivation of the Channel Estimation $\hat{\mathrm{g}}_{1}$}

In order to obtain the channel estimation mismatch, the estimated channel $\hat{g}_{1}$ is developed in the manner of [9], as a Maclaurin series of noise power $\sigma^{2}$.

The following lemma is an important tool in the derivation of the required result.

Lemma 1: [9]. The matrix $\sigma^{2} \mathbf{R}_{\mathbf{y}}^{-1}$ can be expressed as:

$\sigma^{2} \mathbf{R}_{\mathbf{y}}^{-1}=\left[\mathbf{I}-\mathbf{V}_{s} \mathbf{D} \mathbf{V}_{s}^{H}\right]=\mathbf{R}_{0}+\sigma^{2} \mathbf{R}_{1}+\sigma^{4} \mathbf{R}_{2}+\sigma^{6} \mathbf{R}_{3}+O\left(\sigma^{8}\right)$

$$
\begin{gathered}
\mathbf{D}=\boldsymbol{\Lambda}_{s}\left(\boldsymbol{\Lambda}_{s}+\sigma^{2} \mathbf{I}\right)^{-1}, \mathbf{R}_{0}=\mathbf{V}_{n} \mathbf{V}_{n}^{H} \\
\mathbf{R}_{1}=\mathbf{V}_{s} \boldsymbol{\Lambda}_{s}^{-1} \mathbf{V}_{s}^{H}, \mathbf{R}_{2}=-\mathbf{V}_{s} \boldsymbol{\Lambda}_{s}^{-2} \mathbf{V}_{s}^{H}, \mathbf{R}_{3}=\mathbf{V}_{s} \boldsymbol{\Lambda}_{s}^{-3} \mathbf{V}_{s}^{H}
\end{gathered}
$$

where $\boldsymbol{\Lambda}_{s}, \mathbf{V}_{s}$ and $\mathbf{V}_{n}$ are obtained from the eigendecomposition of $\mathbf{R}_{\mathbf{y}}$ given by

$$
\mathbf{R}_{\mathbf{y}}=\left[\begin{array}{ll}
\mathbf{V}_{s} & \mathbf{V}_{n}
\end{array}\right]\left[\begin{array}{cc}
\boldsymbol{\Lambda}_{s} & 0 \\
0 & 0
\end{array}\right]\left[\begin{array}{c}
\mathbf{V}_{s}^{H} \\
\mathbf{V}_{n}^{H}
\end{array}\right]+\sigma^{2} \mathbf{I}
$$

Recalling equation (11) we observe that $\hat{\mathrm{g}}_{1}$ is the least significant generalized eigenvector of the matrix pencil $\left(\mathbf{C}_{1}^{H} \mathbf{R}_{\mathbf{y}}^{-1} \mathbf{R}_{\mathbf{y}}^{-1} \mathbf{C}_{\mathbf{1}}, \mathbf{C}_{1}^{H} \mathbf{R}_{\mathbf{y}}^{-1} \mathbf{C}_{1}\right)$. When the noise power $\sigma^{2}$ tends to zero, the eigenvalues of $\mathbf{R}_{\mathbf{y}}^{-1}$ corresponding to the noise subspace, tend to infinity. Thus, both matrices $\mathbf{C}_{1}^{H} \mathbf{R}_{\mathbf{y}}^{-1} \mathbf{R}_{\mathbf{y}}^{-1} \mathbf{C}_{1}$ and $\mathbf{C}_{1}^{H} \mathbf{R}_{\mathbf{y}}^{-1} \mathbf{C}_{\mathbf{1}}$ suffer from the same pathological problem, which introduces difficulties in obtaining a closed form of $\hat{\mathrm{g}}_{1}$ directly. In order to circumvent this problem, we introduce a modified matrix pencil $\left(\sigma^{4} \mathbf{C}_{1}^{H} \mathbf{R}_{\mathbf{y}}^{-1} \mathbf{R}_{\mathbf{y}}^{-1} \mathbf{C}_{1}, \sigma^{2} \mathbf{C}_{1}^{H} \mathbf{R}_{\mathbf{y}}^{-1} \mathbf{C}_{1}\right)$. According to Lemma 1, as the noise variance tends to zero both entries in this new matrix pencil do not tend to infinity. Moreover, if $\lambda$ denote the generalized eigenvalues of the former matrix pencil and $\mu$ those of the new one then $\mu=\lambda / \sigma^{2}$. Furthermore, the generalized eigenvectors of these two matrix pencils of corresponding eigenvalues are identical. Thus, we can switch our objective to the derivation of the least significant generalized eigenvector and eigenvalue of the new matrix pencil, i.e.

$$
\sigma^{4} \mathbf{C}_{1}^{H} \mathbf{R}_{\mathbf{y}}^{-1} \mathbf{R}_{\mathbf{y}}^{-1} \mathbf{C}_{1} \mathbf{v}=\lambda \sigma^{2} \mathbf{C}_{1}^{H} \mathbf{R}_{\mathbf{y}}^{-1} \mathbf{C}_{1} \mathbf{v} .
$$

Now, we express these two matrices, $\lambda$ and $\mathbf{v}$ as Maclaurin series of $\sigma^{2}$ and substitute them into (12). After arranging both sides as series of $\sigma^{2}$, we can obtain by comparing the coefficients of $\sigma^{2}$ a set of equations with (13) and (14) shown below and (15) and (16) shown at the top of next page.

$$
\begin{aligned}
& \mathbf{A}_{0} \mathbf{v}_{0}=\lambda_{0} \mathbf{B}_{0} \mathbf{v}_{0}, \\
& \mathbf{A}_{0} \mathbf{v}_{1}=\lambda_{1} \mathbf{B}_{0} \mathbf{v}_{0}+\lambda_{0} \mathbf{B}_{1} \mathbf{v}_{0}+\lambda_{0} \mathbf{B}_{0} \mathbf{v}_{0},
\end{aligned}
$$

where

$$
\begin{gathered}
\mathbf{A}_{0}=\mathbf{C}_{1}^{H} \mathbf{R}_{0} \mathbf{C}_{1}, \mathbf{A}_{1}=-\mathbf{C}_{1}^{H} \mathbf{R}_{2} \mathbf{C}_{1}, \mathbf{A}_{2}=-2 \mathbf{C}_{1}^{H} \mathbf{R}_{3} \mathbf{C}_{1}, \\
\mathbf{B}_{k}=\mathbf{C}_{1}^{H} \mathbf{R}_{k} \mathbf{C}_{1}(k=0,1,2,3),
\end{gathered}
$$

and $\lambda_{k}$ and $\mathrm{v}_{k}$ denote the coefficients of $\sigma^{2 k}$ in the expansion.

Although $\mathbf{A}_{0}$ and $\mathbf{B}_{0}$ are actually identical, we use this notation for clarity of presentation. It is seen that (13) represents the asymptotic case $\sigma^{2} \rightarrow 0$. Since $\mu$ is equal to $\lambda / \sigma^{2}$ when $\sigma^{2} \rightarrow 0, \mu$ will tend to infinity unless $\lambda_{0}$ is equal to 0 . We examine now whether such case, i.e. that $\lambda_{0}=0$, is possible, or equivalently whether $\mathbf{C}_{1}^{H} \mathbf{V}_{n} \mathbf{V}_{n}^{H} \mathbf{C}_{1}$ $\left(\mathbf{A}_{0}\right.$ and $\left.\mathbf{B}_{0}\right)$ has a zero eigenvalue and eigenvector. If so, this eigenvalue must be the minimum generalized eigenvalue of the matrix pencil $\left(\mathbf{C}_{1}^{H} \mathbf{R}_{\mathbf{y}}^{-1} \mathbf{R}_{\mathbf{y}}^{-1} \mathbf{C}_{1}, \mathbf{C}_{1}^{H} \mathbf{R}_{\mathbf{y}}^{-1} \mathbf{C}_{1}\right)$ in the ACM algorithm. Clearly, the choice of $\mathbf{v}_{0}=\frac{\mathbf{g}_{1}}{\left\|\mathbf{g}_{1}\right\|}$ qualifies, since $\mathbf{h}=\frac{\mathbf{C}_{1} \mathbf{g}_{1}}{\left\|\mathbf{g}_{\mathbf{1}}\right\|} \in \mathbf{V}_{s}, \mathbf{v}_{0}^{H} \mathbf{C}_{1}^{H} \mathbf{V}_{n} \mathbf{V}_{n}^{H} \mathbf{C}_{1} \mathbf{v}_{0}=0$. Now, we need to examine the conditions under which the solution of $\mathbf{v}_{0}=\frac{\mathbf{g}_{1}}{\left\|\mathbf{g}_{1}\right\|}$ is unique. The following proposition presents a 


$$
\begin{aligned}
\mathbf{A}_{1} \mathbf{v}_{0}+\mathbf{A}_{0} \mathbf{v}_{2}= & \lambda_{0} \mathbf{B}_{0} \mathbf{v}_{2}+\lambda_{1} \mathbf{B}_{0} \mathbf{v}_{1}+\lambda_{0} \mathbf{B}_{1} \mathbf{v}_{1}+\lambda_{0} \mathbf{B}_{2} \mathbf{v}_{0}+\lambda_{2} \mathbf{B}_{0} \mathbf{v}_{0}+\lambda_{1} \mathbf{B}_{1} \mathbf{v}_{0} \\
\mathbf{A}_{0} \mathbf{v}_{3}+\mathbf{A}_{1} \mathbf{v}_{1}+\mathbf{A}_{2} \mathbf{v}_{\mathbf{0}}= & \lambda_{0} \mathbf{B}_{0} \mathbf{v}_{3}+\lambda_{1} \mathbf{B}_{0} \mathbf{v}_{2}+\lambda_{0} \mathbf{B}_{1} \mathbf{v}_{2}+\lambda_{0} \mathbf{B}_{2} \mathbf{v}_{1}+\lambda_{2} \mathbf{B}_{0} \mathbf{v}_{1}+\lambda_{1} \mathbf{B}_{1} \mathbf{v}_{1}+ \\
& \lambda_{0} \mathbf{B}_{3} \mathbf{v}_{0}+\lambda_{1} \mathbf{B}_{2} \mathbf{v}_{0}+\lambda_{2} \mathbf{B}_{1} \mathbf{v}_{0}+\lambda_{3} \mathbf{B}_{0} \mathbf{v}_{0}
\end{aligned}
$$

sufficient condition that guarantees the unique identifiability to within a scale ambiguity.

Proposition 1 (Condition of Identifiability): Under the assumption $\operatorname{dim}\left\{\operatorname{span}\left\{\mathbf{C}_{1}\right\} \bigcap \operatorname{span}\left\{\mathbf{h}_{1,0} \mathbf{H}_{M A I}\right\}\right\}=1$, the solution of $\mathbf{v}_{0}=\frac{\mathbf{g}_{1}}{\left\|\mathbf{g}_{1}\right\|}$ as the zero eigenvector of $\mathbf{C}_{1}^{H} \mathbf{V}_{n} \mathbf{V}_{n}^{H} \mathbf{C}_{1}$ is unique within a scale ambiguity. The symbols $\operatorname{dim}\{\mathbf{X}\}$ and $\operatorname{span}\{\mathbf{X}\}$ denote the dimension and range space of column vectors in $\mathbf{X}$ respectively.

The proofs of all propositions are omitted in this paper due to limited space. The above assumption is general and in line with accepted form as in [6], [7] and [9]. On combining Proposition 1 and the results on the above discussion concerning (13) we obtain a unique solution of $\mathbf{v}_{0}$ and $\lambda_{0}$ as

$$
\lambda_{0}=0, \quad \mathbf{v}_{0}=\frac{\mathbf{g}_{1}}{\left\|\mathbf{g}_{1}\right\|} .
$$

In the following proposition, we present $\hat{\mathrm{g}}_{1}$ as Maclaurin series when $\sigma^{2}$ is around 0 . The higher order entries in $\sigma^{2}$ in the expansion of $\hat{g}_{1}$ represent the estimation mismatch.

Proposition 2: The minimum generalized eigenvector of matrix pencil $\left(\mathbf{C}_{1}^{H} \mathbf{R}_{\mathbf{y}}^{-1} \mathbf{R}_{\mathbf{y}}^{-1} \mathbf{C}_{1}, \mathbf{C}_{1}^{H} \mathbf{R}_{\mathbf{y}}^{-1} \mathbf{C}_{1}\right)$ are given by

$$
\hat{\mathbf{g}}_{1}=\mathbf{v}_{0}+\sigma^{4} \mathbf{v}_{2}+O\left(\sigma^{6}\right) \text {, }
$$

where

$$
\mathbf{v}_{0}=\frac{\mathbf{g}_{1}}{\left\|\mathbf{g}_{1}\right\|}, \mathbf{v}_{2}=\mathbf{A}_{0}^{\dagger}\left(\frac{\mathbf{g}_{1}^{H} \mathbf{A}_{1} \mathbf{g}_{1}}{\mathbf{g}_{1}^{H} \mathbf{B}_{1} \mathbf{g}_{1}} \mathbf{B}_{1}-\mathbf{A}_{1}\right) \frac{\mathbf{g}_{\mathbf{1}}}{\left\|\mathbf{g}_{1}\right\|},
$$

and the symbol $\dagger$ denotes Moore-Penrose generalized inverse.

According to (18) when the noise power $\sigma^{2}$ tends to 0 , the channel of user 1 can be estimated without error provided that the identifiability condition holds. Furthermore, in a noisy environment, under the above conditions the estimation mismatch is very small, because the most significant mismatch is on the order of $\sigma^{4}$. This point guarantees the performance of the ACM algorithm over a wide range of noise power levels. In [9] the authors also investigate the estimation mismatch of the MV algorithm given by (46) in [9] and reproduced below

$$
\hat{\mathbf{g}}_{1, M V}=\frac{\mathbf{g}_{1}}{\left\|\mathbf{g}_{1}\right\|}-\sigma^{2} \mathbf{B}_{0}^{\dagger} \mathbf{B}_{1} \frac{\mathbf{g}_{1}}{\left\|\mathbf{g}_{\mathbf{1}}\right\|}+O\left(\sigma^{4}\right) .
$$

Clearly in the MV case the estimation mismatch has a component of the order of $\sigma^{2}$ and thus is much larger than the proposed ACM case. As far as computational complexity is concerned it is observed that the MV algorithm requires the calculation of the least significant eigenvector of matrix $\mathbf{C}_{1}^{H} \mathbf{R}_{\mathbf{y}}^{-1} \mathbf{C}_{1}$, while the algorithm proposed in this paper requires the solution of a generalized eigenproblem of the same size. However, since both $\mathbf{C}_{1}^{H} \mathbf{R}_{\mathbf{y}}^{-1} \mathbf{R}_{\mathbf{y}}^{-1} \mathbf{C}_{1}$ and $\mathbf{C}_{1}^{H} \mathbf{R}_{\mathbf{y}}^{-1} \mathbf{C}_{1}$ have much smaller sizes than $\mathbf{R}_{\mathbf{y}}^{-1}$, the increase in computational complexity is not significant.

\section{B. SINR Comparison}

In the following proposition we present the limit of $S I N R$ ratio between the MMSE and ACM algorithms as the noise power $\sigma^{2}$ tends to 0 .

Proposition 3: The limit of the ratio of $S I N R_{M M S E}$ and $S I N R_{A C M}$ is given by

$$
\lim _{\sigma^{2} \rightarrow 0} \frac{S I N R_{M M S E}}{S I N R_{A C M}}=1 .
$$

The above ratio indicates that as the noise power $\sigma^{2}$ tends to zero, the SINR of the ACM algorithm and that of the MMSE algorithm converge at the same rate to infinity. Since the MMSE algorithm is optimal in the sense that it maximizes the SINR, clearly the SINR of any blind algorithm must be less than this case for a given noise level. It follows from proposition 3 that the ACM algorithm exhibits optimal performance in the asymptotic sense. A further point to observe is the following. If we examine the corresponding ratio for the MMSE and the MV algorithms as given in [9] and reproduced below

$$
\lim _{\sigma^{2} \rightarrow 0} \frac{S I N R_{M M S E}}{S I N R_{M V}}=1-\frac{\mathbf{g}_{1}^{H} \mathbf{B}_{1} \mathbf{B}_{0}^{\dagger} \mathbf{B}_{1} \mathbf{g}_{1}}{\mathbf{g}_{1}^{H} \mathbf{B}_{2} \mathbf{g}_{1}},
$$

we note that the second term in (20) is negative definite and hence this ratio is strictly larger than 1 . Thus, it is evident that the ACM algorithm exhibits better performance than the MV algorithm.

\section{Simulation Studies}

In this section, we use Monte Carlo computer simulations to compare the performances of the proposed algorithm with the MV and MMSE algorithms. Consider an asynchronous CDMA system using Gold codes of length 31 and each user's signal transmitting through a different multipath channel of delay spread equal to 4 chips. The delays of interfering users, $\tau_{k}(k=2, \ldots, K)$ are assumed to be uniformly distributed between 0 and $T_{s}=31 T_{c}$. We choose an observation window of duration up to 40 chips. This implies that the receiver has been coarsely synchronized with the desired user (user 1) and that it tolerates a synchronization error $\tau_{1}$ restricted to be between 0 and $5 T_{c}$. The power advantage of interfering users with respect to the desired user is denoted as interference to signal ratio (ISR) given by $I S R=\frac{\left\langle h_{k}^{2}(t)\right\rangle}{\left\langle h_{1}^{2}(t)\right\rangle},(k=2, \ldots, K)$ where $\left\langle h^{2}(t)>\right.$ represents the energy of the signal. We simulate a severe near-far resistant case in which the desired user has a weak power of $20 \mathrm{~dB}$ less than interfering users, i.e. $I S R=20 d B$. The input signal to noise ratio (SNR) of the desired user is defined as $S N R=\frac{\left\langle h_{1}^{2}(t)\right\rangle}{\sigma^{2}}$. All plots are 
averaged over 50 independent runs of random generation of multipath channel, synchronization error of the desired user and delays of all interfering users. In Fig. 1, the system output SINR versus number of active users where $S N R=5 d B$ and over sampling factor $M=1$ is shown for comparison of the following three algorithms:

- Proposed ACM algorithm in (11);

- MMSE algorithm which uses the information of channel state as a prior knowledge;

- A modified version of MV algorithm proposed in [9], where a modification is made to consider the effect of synchronization errors.

It is seen that the ACM algorithm produces an improved performance over the modified MV algorithm especially when the number of active users is close to the system capacity, (13 in the case under consideration). In addition, the ACM algorithm shows a performance almost identical to the MMSE algorithm before the number of active users exceeds this capacity threshold. Beyond that point both ACM and MV algorithm suffer severe performance degradation since the condition of identifiability no longer holds and the channel can no longer be accurately estimated.

Fig. 2 shows the system output SINR versus the input SNR of the desired user where the number of active users is 10 and over sampling factor $M=1$. There is a $3 \mathrm{~dB}$ SINR gap between the MV algorithm and the ACM algorithm, in favor of the latter case, over the complete range of noise power level, while the performances of the ACM and MMSE algorithms are almost identical. Fig. 3 further confirms the result of Proposition 3 by showing the SINR ratios between the MMSE, ACM and MV algorithms (Note that no logarithm operation is involved in the calculation of the SINR ratio). It is noted that while $\frac{S I N R_{M M S E}}{S I N R A S M}$ converges to 1 with the increase of the input SNR, $\frac{S I N R_{M M S E}}{S I N R M V}$ fluctuates close to 2, which exactly coincides with the $3 \mathrm{~dB}$ gap shown in Fig. 2 .

\section{ConClusions}

In this paper the problem of joint channel estimation and interference suppression in asynchronous CDMA systems over frequency-selective fading channels has been considered. We adopted a system model that includes aspects of synchronization error and multipath delay spread overestimation, both of which are of significant practical interests in receiver design. Our analytical approach yields an important SINR ratio limit between the MMSE and ACM algorithm, which indicates that the ACM algorithm provides an asymptotic optimal performance in the sense of maximizing output SINR.

\section{REFERENCES}

[1] F. Adachi, M. Sawahashi, and H. Suda, "Wideband DS-CDMA for nextgeneration mobile communications systems:" IEEE Personal Commun., vol. 36 , pp. 56-69, Sept. 1998.

[2] S. Verdu, Multiuser Detection. Cambrdige U.K.: Cambridge University Press, 1998

[3] M. Honig, U. Madhow, and S. Verdu, "Blind adaptive multiuser detection," IEEE Trans. Inform. Theory, vol. 41, pp. 944-960, July 1995.

[4] X. Wang and H. V. Poor, "Blind multiuser detection: a subspace appraoch," IEEE Trans. Inform. Theory, vol. 44, pp. 677-690, Mar. 1998.
[5] R. O. LaMaire, A. Krishna, P. Bhagwat, and J. Panian, "Wireless LANs and mobile networking: standards and future directions," IEEE Commun. Mag., vol. 34, pp. $86-94$, Aug. 1996.

[6] H. Liu and G. Xu, "A subspace method for signiture waveform estimation in synchronous CDMA systems," IEEE Trans. Commun., vol. 44, pp. 1346-1354, Oct. 1996.

[7] M. Torlak and G. Xu, "Blind multiuser channel estimation in asynchronous CDMA systems," IEEE Trans. Signal Processing, vol. 45, pp. 137-147, Jan. 1997.

[8] S. Bensley and B. Aazhang, "Subspace-based channel estimation for code division multiple access communication systems," IEEE Trans. Commun., vol. 44, pp. 1009-1020, Aug. 1996.

[9] M. K. Tsatsanis and $\mathrm{Z}$. Xu, "Performance analysis of minimum variance CDMA receivers," IEEE Trans. Signal Processing, vol. 46, pp. 30143022, Nov. 1998.

[10] S. Zhu, P. Arasaratnam, and A. G. Constantinides, "A new family of blind CDMA receivers based on second order statistics," in Proc. Globecom' 02 , pp. $448-452$, Nov. 2002.

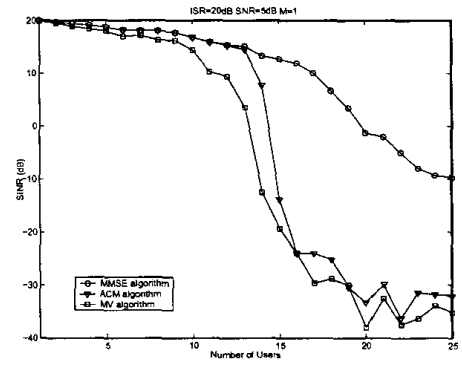

Fig. 1. SINR of MMSE, ACM and MV algorithms versus number of users System parameter: $I S R=20 \mathrm{~dB}, S N R=5 \mathrm{~dB}$ and $\mathrm{M}=1$

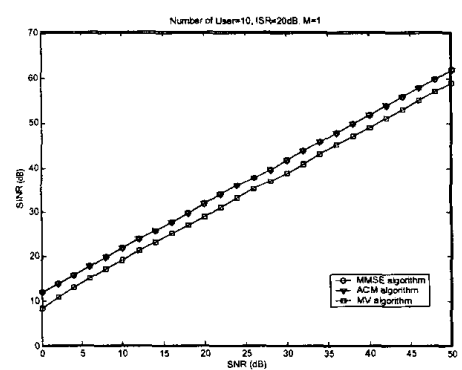

Fig. 2. SINR of MMSE, ACM and MV algorithms versus SNR of user 1 System parameter: $\mathrm{ISR}=20 \mathrm{~dB}$, Number of user $=10$ and $\mathrm{M}=1$
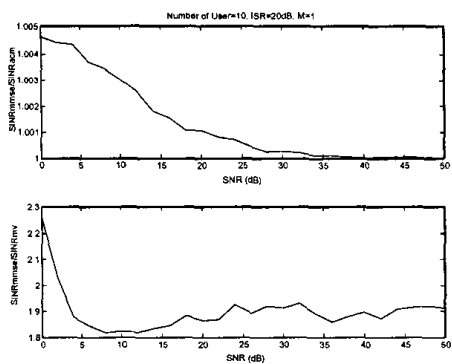

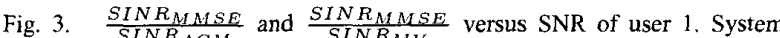
parameter: ISR=20dB, Number of user $=Y_{0}$ and $\mathrm{M}=1$ 